\title{
Laberintectomía quirúrgica
}

\author{
Surgical Labyrinthectomy
}

Natalia Cabrera $\mathbf{S}^{1}$, Luis Dentone $\mathbf{S}^{1}$, Carlos Stott $\mathbf{C}^{1}$, Paul H. Délano $\mathbf{R}^{1,2}$.

\begin{abstract}
RESUMEN
El tratamiento quirúrgico del vértigo se puede dividir en dos grandes grupos: no ablativos y ablativos, dentro de éstos últimos se encuentra la laberintectomía quirúrgica, técnica con la cual se produce cofosis. Su principal indicación es la sintomatología vestibular periférica invalidante, unilateral, que no mejora con medidas farmacológicas ni con terapia psiquiátrica, asociado a hipoacusia sensorioneural severa a profunda. Existen dos tipos de abordajes para realizar la laberintectomía quirúrgica: transmastoidea y transcanal, siendo la primera el gold standard para eliminar la función vestibular. Se presentarán dos casos clínicos de pacientes sometidos a estos procedimientos, uno por vía transmastoidea y otro por vía transcanal.

Palabras clave: Enfermedad de Ménière, vértigo, desequilibrio, laberintectomía, gentamicina.
\end{abstract}

\begin{abstract}
The surgical treatment of vertigo can be classified into two main groups: non-ablative and ablative. The surgical labyrinthectomy is an ablative treatment in which no hearing is preserved. Its main indication is the disabling peripherical and unilateral vestibular symptoms that do not improve with pharmacological measures or with psychiatric therapy, in patients with severe to profound sensorineural hearing loss. There are two types of approaches for labyrinthectomy, transmastoid and transcanal, the first is the gold standard to eliminate the vestibular function. Here, we present two cases of patients undergoing these procedures, one by transmastoid approach and the other by transcanal approach.

Key words: Ménière disease, vertigo, disequilibrium, labyrinthectomy, gentamicin.

\section{INTRODUCCIÓN}

para el tratamiento del vértigo infrecuentes. Diez a $15 \%$ de los pacientes con enfermedad de Ménière (EM) son refractarios a tratamiento médico, y constituyen la causa más frecuente para indicar un tratamiento invasivo, incluyendo inyecciones
\end{abstract}

El tratamiento médico del vértigo y desequilibrio mejora la sintomatología de la gran mayoría de los pacientes, siendo las indicaciones quirúrgicas

\footnotetext{
1 Departamento Otorrinolaringología, Hospital Clínico de la Universidad de Chile.

2 Programa de Fisiología y Biofísica, ICBM, Facultad de Medicina, Universidad de Chile.
}

Recibido el 11 de diciembre, 2017. Aceptado el 17 de abril, 2017. 
transtimpánicas de gentamicina 0 tratamientos quirúrgicos ${ }^{1}$. Otras indicaciones menos frecuentes de tratamiento quirúrgico del vértigo corresponden a la dehiscencia del canal semicircular superior, vértigo paroxístico postural y fístula perilinfática, entre otros.

El tratamiento quirúrgico del vértigo se puede dividir en dos grandes grupos: no ablativos y ablativos (Figura 1). Dentro de las técnicas no ablativas se encuentran el drenaje del saco endolinfático, oclusión de canales semicirculares y laberintectomía química con gentamicina. Dentro de los métodos ablativos, existen aquellos que preservan la audición (ablativo selectivo) como la neurectomía singular y neurectomía vestibular (retrosigmoídea-retrolaberíntica-fosa media) y aquellos que destruyen el oído interno y dejan al paciente sin audición (ablativo no selectivo), como la neurectomía vestibular translaberíntica y la laberintectomía quirúrgica².

La laberintectomía quirúrgica, es un procedimiento efectivo para el manejo de la disfunción vestibular periférica no compensada unilateral, que se ofrece como una medida de rescate en un paciente con hipoacusia severa a profunda. Consiste en la apertura del vestíbulo y canales semicirculares (CSC) para eliminar por completo a los receptores y aferencias vestibulares ${ }^{3}$. La laberintectomía quirúrgica se indica en caso de síntomas vestibulares periféricos persistentes e invalidantes, que no mejoran con medidas farmacológicas ni con terapia psiquiátrica, asociado a hipoacusia sensorioneural severa a profunda y que afecta a un solo oído. De esta forma, esta cirugía está contraindicada si se trata de un paciente con oído único ${ }^{4}$. La laberintectomía provoca una desaferentación completa de los receptores vestibulares que puede ser manejada en forma satisfactoria con rehabilitación vestibular.

Desde el punto de vista de quirúrgico, existen dos tipos de abordajes para realizar la laberintectomía: transmastoidea y transcanal2. El abordaje transmastoideo es el gold standard para la remoción completa de todos los elementos neuroepiteliales del oído interno. Permite la visualización completa del vestíbulo y remoción de utrículo, sáculo y ampollas de los CSC2${ }^{2}$. Entre sus ventajas sobre la técnica transcanal, se encuentra menor desequilibrio poscirugía (22\% vs $62 \%)^{5}$ y mayor tasa de mejoría de la calidad de vida $(98 \%)^{6}$. Respecto a la técnica del abordaje transcanal, en este se confecciona un colgajo tímpano-meatal y se accede a la caja timpánica, removiendo el estribo de la ventana oval. Luego se realiza un fresado entre las ventanas oval y redonda, accediendo al vestíbulo y eliminando los componentes membranosos del utrículo y sáculo con un gancho en $90^{\circ 3}$. Con la técnica transcanal se logra un tratamiento efectivo del vértigo en el $80 \%-100 \%{ }^{5}$, pero con inestabilidad residual en $60 \%$ los pacientes (cifra

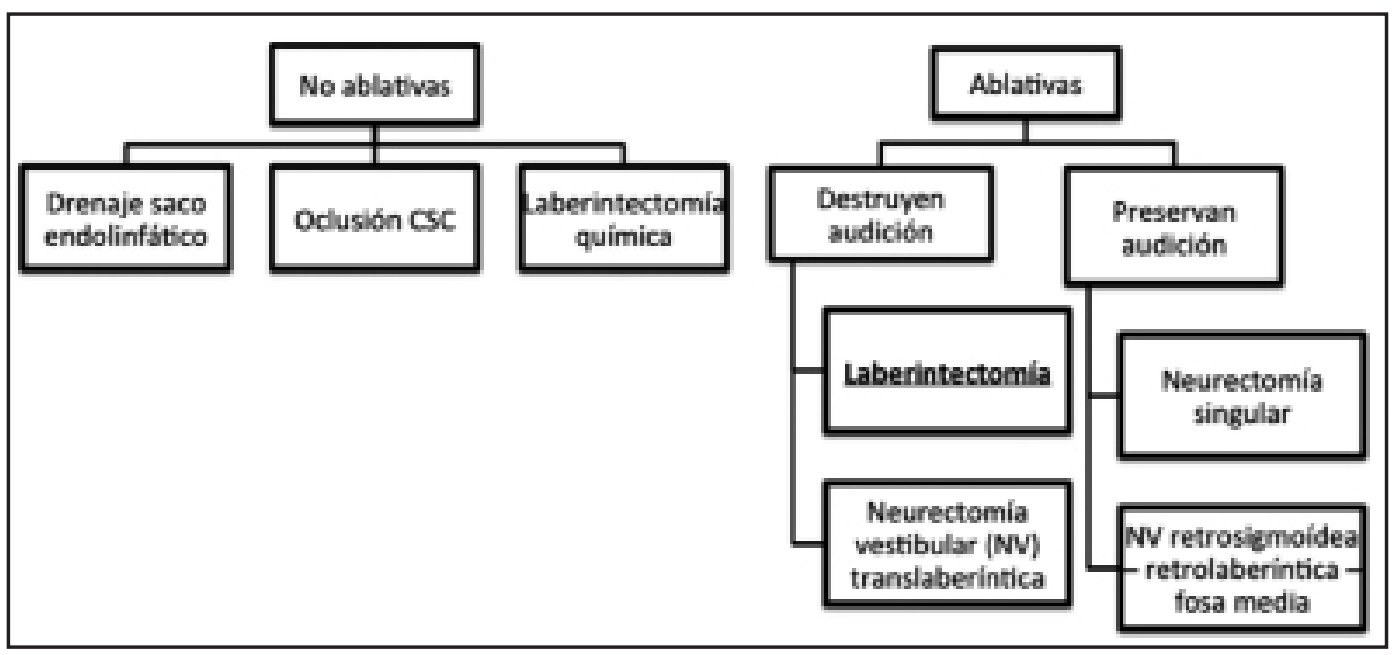

Figura 1. Tratamiento de vértigo refractario. 
3 veces superior a la transmastoidea) ${ }^{3}$. Entre sus ventajas destaca ser un procedimiento menos invasivo con un menor tiempo operatorio y con menos morbilidad. Entre las desventajas de la técnica transcanal, se encuentra que la exposición quirúrgica es limitada, tiene mayor dificultad en la visualización de todos los elementos neuroepiteliales del sistema vestibular y una mayor tasa de laberintectomía incompleta ${ }^{7}$.

A continuación se presentan dos casos de pacientes con vértigo refractario a tratamiento médico, en los que se realizó una laberintectomía quirúrgica, pertenecientes a la casuística del Hospital Clínico de la Universidad de Chile ( $\mathrm{HCUCH})$. Los pacientes aprobaron un consentimiento informado.

\section{Caso 1}

Hombre de 52 años, operador de camiones pesados con antecedente de cirugía radical del oído izquierdo en 1997, que evoluciona con vértigo persistente e invalidante, requiriendo licencia laboral por más de 1 año. Recibió diversos tratamientos médicos sin resultados, incluyendo rehabilitación vestibular, carbamazepina indicada por neurología y gentamicina transtimpánica. El examen de VIII par muestra un oído derecho con audición normal (PTP de 15 dB HL y discriminación $100 \%$ ), mientras que el oído izquierdo tiene una hipoacusia mixta (PTP 93/48 dB HL) con mala discriminación (16\%) a 100 dB e hipoexcitabilidad vestibular del oído izquierdo de $50,1 \%$ según frecuencia. No presentaba nistagmo espontáneo. RNM cerebro con énfasis en fosa posterior sin hallazgos patológicos. Con diagnóstico de vértigo invalidante persistente es derivado al Comité de Oído del HCUCH donde, dado el antecedente de cirugía radical previa, se le ofrece al paciente laberintectomía transmastoidea del oído izquierdo. Se realizó laberintectomía transmastoidea, identificando dehiscencia del canal lateral, sitio donde se inició el fresado de los canales lateral y posterior, accediendo al utrículo, y aspirando los componentes membranosos del laberinto. Se colocó relleno con grasa para obliterar los canales y el vestíbulo e injerto de periostio en la cavidad radical. En el posoperatorio el paciente evolucionó sin nistagmo, pero con desequilibrio de la marcha que se manejó con rehabilitación vestibular. El paciente fue controlado a los 6 meses, logrando la reinserción laboral, pero con sordera completa del oído izquierdo.

\section{Caso 2}

Mujer de 48 años con síndrome de Ménière diagnosticado a los 19 años, cursa durante el último año crisis de vértigo diarias, asociadas a baja de peso de $22 \mathrm{~kg}$. Se realiza tratamiento con gentamicina intratimpánica sin mejoría de vértigo, y evoluciona con anacusia del oído izquierdo y es derivada para laberintectomía quirúrgica. Se realiza VIII par que muestra en oído derecho PTP de 8,3 dB y discriminación $100 \%$. En oído izquierdo no tiene umbrales y con discriminación $0 \%$. Presenta hipoexcitabilidad vestibular de $88 \%$ del oído izquierdo, según la velocidad de la componente lenta de la videooculografía y con un nistagmo espontáneo grado III con fase rápida a izquierda. Se realiza estudio de hidrops secundario, destacando un examen de anticuerpos-antinucleares (ANA) positivo 1/320, sugerente de lupus eritematoso sistémico. La paciente fue derivada a reumatología y se inicia tratamiento con metilprednisolona, el cual no logró disminuir la sintomatología vertiginosa. Reumatología solicita laberintectomía quirúrgica del oído izquierdo. Al tratarse de un oído sin cirugías previas, se decide realizar una laberintectomía transcanal. Se levanta un colgajo tímpano-meatal de las 12 a las 7 según los punteros del reloj, desplazando la cuerda del tímpano, y luego se rebaja la pared póstero-superior. Se identifica articulación incudo-estapedial y se realiza una estapedectomía incluyendo la platina. Luego se realiza un fresado entre la ventana oval y redonda y se rebaja el nicho de la ventana redonda hasta llegar al nervio singular que se secciona. Luego se utilizó un gancho de $90^{\circ}$ en el vestíbulo para remover las ampollas de los CSC y se aspira el contenido membranoso. Se coloca gelfoam con gentamicina en vestíbulo. Luego se reposiciona el colgajo tímpano-meatal. La paciente evoluciona en forma favorable, con inversión del nistagmo y se indica rehabilitación vestibular. A los 6 meses la paciente se encuentra con un leve desequilibrio, pero sin vértigo. 


\section{DISCUSIÓN}

Indicar una laberintectomía quirúrgica no es una decisión simple, por lo que ésta debe ser realizada en el contexto de un Comité de Oído por varios otorrinolaringólogos. Es importante destacar que la ausencia de respuesta auditivas en una audiometría, como en el caso $n^{0} 2$, no implica ausencia de respuestas vestibulares, y si el paciente presenta sintomatología vestibular atribuible al oído cofótico, se puede indicar una laberintectomía quirúrgica.

Existen alternativas previas a la indicación de una laberintectomía quirúrgica, como la gentamicina transtimpánica, la que debe ser siempre considerada antes de decidir una laberintectomía quirúrgica. Más aún, muchas veces los pacientes con enfermedad de Ménière no han sido estudiados en forma completa, como por ejemplo en nuestro caso clínico $n^{0} 2$, donde no se había solicitado estudio de enfermedades autoinmunes, y se encontró un examen (ANA (+)) sugerente de lupus eritematoso sistémico, una enfermedad que puede provocar hidrops endolinfático ${ }^{8}$. Además de las enfermedades autoinmunes, es importante descartar una otosífilis, ya que puede provocar un hidrops secundario que suele ser bilateral ${ }^{9}$. Por otra parte, es conocido que muchos de los pacientes con Ménière, tienen también patología psiquiátrica asociada, por lo que recomendamos que todo paciente candidato a una laberintectomia química o quirúrgica sea evaluado previamente por un especialista en salud mental ${ }^{10}$.

\section{BIBLIOGRAFÍA}

1. Sismanis A. Surgical management of common peripheral vestibular diseases. Otorhinolaryngologia - Head and Neck Surgery 2010; 40: 10-5.

2. TeUfert K, DoherTy J. Endolymphatic Sac Shunt, Labyrinthectomy, and Vestibular Nerve Section in Meniere's Disease. Otolaryngol Clin N Am 2010; 43: 1091-111.

3. TELIAN S. Surgery for vestibular disorders. En: Cummings C, Flint P. Cummings Otolaryngology Head \& Neck Surgery. Fifth edition. Philadelphia, PA: Mosby/Elsevier, 2010.
En relación a las diferencias entre laberintectomía transcanal y transmastoidea, debido a que existe poca literatura, no hay evidencia concluyente. Sugerimos utilizar como primera opción al abordaje transcanal, ya que es menos invasivo, con menor tiempo operatorio y con similar resultado funcional. Sin embargo, esta decisión debe ser tomada caso a caso y considerar las posibles alteraciones anatómicas de una cirugía previa de oído ${ }^{7}$.

Los pacientes que se someten a una laberintectomía quirúrgica, deben saber que su recuperación no será rápida, y más aún que requerirán de rehabilitación vestibular por meses 0 incluso de por vida. Más aún, a pesar de este tratamiento, muchas veces los pacientes requieren cambiar su actividad laboral, ya que puede existir hasta en $30 \%$ de los casos un desequilibrio persistente ${ }^{5}$. Los pacientes también deben conocer que posterior a la cirugía quedarán con sodera completa del oído operado. Sin embargo, la cofosis posoperatoria del oído al que se le realizó la laberintectomía, es un problema que podría ser resuelto con un implante coclear colocado en la misma cirugía ${ }^{11} 0$ en un segundo tiempo quirúrgico ${ }^{12}$. Más aún, en el futuro, es probable que se pueda recuperar la función vestibular, ya que se están desarrollando implantes vestibulares en seres humanos ${ }^{13}$.

En conclusión la laberintectomía quirúrgica, transcanal 0 transmastoidea, son opciones de rescate en el paciente con vértigo crónico. En estos casos seleccionados, la laberintectomía quirúrgica reduce la sintomatología vestibular y mejora la calidad de vida de los pacientes.

4. Coker NJ. Jenkins HA. Labyrinthectomy. En: Coker NJ, Jenkins HA. Atlas of otologic surgery. Philadelphia: WB Saunders company; 2001.

5. Langman AW, Lindeman RC. Surgical labyrinthectomy in the older patient. Otolaryngol Head Neck Surg 1998; 118(6): 739-42.

6. Díaz R, LaRouere M, Bojrab D, Zappia J, Sargent E, ShaIA W. Quality-of-Life Assessment of Ménière's Disease Patients After Surgical Labyrinthectomy. Otol \& Neurotol 2007; 28: 74-86.

7. Dickins JRE, Graham SS. Surgical treatment of peripheral vestibular disorders. En: Glasscock ME, Gulya AJ. Glasscock-Shambaug's Surgery 
of the ear. Fifth edition. Ontario: BC Deckers, 2005.

8. Batuecas A, Del Pino J, Cordero C, Calle MI, López $J$. Hearing and vestibular disorders in patients with systemic lupus erythematosus. Lupus 2013; 22(5): 437-42.

9. Phillips JS, Gaunt A, Phillips DR. Otosyphilis: a neglected diagnosis? Otol Neurotol2014; 35(6): 1011-3.

10. Lahmann $\mathrm{C}$, Henningsen P, Brandt T, Strupp M, Jahn K, Dieterich M, Eckhardt-Henn A, Feuerecker R, Dinkel A, Schmid G. Psychiatric comorbidity and psychosocial impairment among patients with vertigo and dizziness. $J$ Neurol Neurosurg Psychiatry 2015; 86(3): 302-8.

11. Doobe G, Ernst A, Ramalingam R, Mittmann P, Todt I. Simultaneous labyrinthectomy and cochlear implantation for patients with single-sided Ménière's disease and profound sensorineural hearing loss. Biomed Res Int 2015; 2015: ID 457318.

12. Osborn HA, Yeung R, Lin VY. Delayed cochlear implantation after surgical labyrinthectomy. $J$ Laringol Otol 2012; 126(1): 63-5.

13. Aedo-Sánchez C, Délano, PH. Implantes vestibulares. Rev Otorrinolaringol y Cirugía de Cabeza y Cuello 2013; 73(3): 271-5. 